\title{
Internet Services for Powder Diffractionists
}

The Internet has grown to more than 32000 individual networks connecting well over 1 million computers as of 1994, and it is still growing exponentially. Usage has grown at the rate of about $11 \%$ per month for the last 5 years. One year ago it was difficult to find a book about the Internet, and this year our local computer store devotes four 6-ft-long shelves to that single subject. A vast wealth of information is available on the Internet, and the software to help find and retrieve the required information is improving. There are bulletin boards and Usenet news groups such as sci.techniques.xtallography [see Powder Diffr. 9, 1 (March 1994)] targeted toward powder diffraction.

The proliferation of Internet connections in industry and academic institutions throughout the world makes it increasingly likely that your colleagues are attached to the net. It is estimated that there are more than 32 million electronic mail (e-mail) users in the United States. E-mail has become an indispensable part of doing business at the ICDD. In addition to sending messages, e-mail can be used to join discussion lists, called mailing lists, on specific subjects, similar to Usenet, but each posting is sent directly to you. Mailing lists allow those people without access to Usenet to participate in discussion groups. Three groups that might be of interest include the Chemical Information Sources List, CHEMINF-L (subscribe by sending mail to listserv@iubvm.bitnet, which contains the following command in the message body: SUBSCRIBE CHEMINF-L your-first-name your-last-name), the Computational Chemistry List (to get more information, send a short note stating your name, affiliation, and e-mail address to: chemistry-request@oscsunb.osc.edu), and the Rietveld Mail List (subscribe by sending mail to rietveld_subscribe@xtal.cmc.uab.edu). Make sure that you understand the "culture" of the mailing list before you send messages to it. You might want to be conservative in joining mailing lists because you will receive considerable material (some lists generate in excess of 100 messages per day).

Bulletin boards are accessed by anonymous File Transfer Protocol (FTP) or by the free navigational aids mentioned later. Bulletin boards of interest include Alfred University (anonymous FTP to xray.alfred.com (149.84.68.2)), CONCISE (telnet to concise.level-7.co.ak, log in as concise, use concise as the password, look in /sigs/crystal for information), ICDD (anonymous FTP to icdd.com (192.102.247.104)), and CSIRO (anonymous FTP to sol.dmp.csiro.au (192.68.132.2), look in /pub/xtallography).

Organizations are joining the Internet to promote the exchange of information. The Mineralogical Society of America (MSA) has established a list server for MSA members at msa-request@smith.edu. The Protein Data Bank (PDB) has both a mailing list server at fileserv@pb1.pdb.bnl.gov (send INFO YOUR-E-MAIL-
ADDRESS in the message body), and access by anonymous FTP at pdb.pdb.bnl.gov (130.199.144.1). The American Institute of Physics maintains PINET, a chargeable service, with a wealth of information. Information about PINET is available from elecprod@aip.org. NASA has been placing space images on the Internet for some time now. Try anonymous FTP to marvel.stsci.edu and look in /stsci/epa/comet for images of Shoemaker-Levy 9's impact on Jupiter.

What promises to be the most useful to those looking for information on the Internet are the free navigational aids called Archie, Gopher, and the World Wide Web.

Archie is useful when you need to search for a specific file or program name. To access Archie, telnet to an Archie server: for example, archie.rutgers.edu (128.6.18.15). Archie servers are normally slow and may not accept new users if they are overloaded. Begin a search for a file by typing prog filename, for example, prog undecode, to find the uudecode program.

Gopher, named after the mascot of the University of Minnesota where Gopher was written, uses menus to search for information. To try it, telnet to a Gopher server, i.e., consultant.micro.umn.edu (134.84.132.4), and $\log$ in as gopher. Gopher sites relevant to crystallography include CSIRO: commsun.its.csiro.au (152.83.8.2), University of Alabama: xtal.cmc.uab.edu (138.26.45.1), and IUCr: gopheriucrac.uk (192.70.242.1). The CSIRO gopher includes the ability to search for e-mail addresses of crystallographers.

The World Wide Web (WWW) is the newest and the best service to use when Archie and Gopher are not applicable. It allows hypertext searching and is very easy to use. With the right software, such as Mosaic, text displayed on the screen looks like a page out of a book, and images are automatically retrieved and placed on the page when a page is downloaded and displayed. Mosaic is available free by anonymous FTP from ftp.ncsa.uiuc.edu (141.142.20.50) for IBM-PC, Macintosh, and X-Windows clients. Without Mosaic, telnet to a WWW server, hnsource.cc.ukans.edu (129.237.33.1), for example. WWW information relevant to crystallography can be found at: http://www.unige.ch/crystal/ crystal_index.html, http://xray.uu.se/, and http://www.iucrac.uk/welcome.html.

There is no better time than now to find out how the Internet services can help you do your job better and faster. In future issues, Computer Comments will cover in greater depth each aspect of the Internet mentioned here.

Mark A. Holomany The International Centre for Diffraction Data Newtown Square, Pennsylvania 